\section{Was den Zeitaufwand für die Operation des Katarakts und des Glaukoms betrifft ${ }^{1}$}

X. Martin

"Messen Sie, wieviel Zeit ich brauche, meine Herren, messen Sie!» würde der berühmte Robert Liston ${ }^{2}$ seinen Studenten zugerufen haben [1]. In der Tat musste zur Zeit von Robert Liston jede Operation schnell vor sich gehen. Die Technik der Anästhesie war noch in ihren Anfängen, und die Keimfreiheit gab es eigentlich noch nicht. So dauerte eine Operation des Katarakts nach der extrakapsulären Technik von Daviel [2] weniger als 10 Minuten, was für einen nicht anästhesierten Patienten schon sehr lange war.

Die Technik der Reklination (Abb. 1), die darin besteht, dass man die Linse in den Glaskörper schiebt, wurde im Fernen Osten bis ins 20. Jahrhundert praktiziert [3]. Diese Handhabung dauerte 1-2 Minuten, wenn sie mit einem einzigen scharfen Instrument ausgeführt wurde, und 2-3 Minuten, wenn man ein scharfes Instrument benutzte, um das Auge zu durchstecken, und ein stumpfes Instrument, um die Linse zurückzuschieben, ohne die Linsenkapsel zu verletzen.

Letzthin ist in der Presse eine Nachricht verbreitet worden, wonach die Operationen des Katarakts und des grünen Stars zu teuer seien, da die moderne Technik es heute ermögliche, diese Operationen viel leichter und schneller auszuführen als früher.

Der Autor dieser Zeilen (XM) hat das Privilegium gehabt, seine chirurgische Ausbildung im Kreuzpunkt der drei Operationstechniken des Katarakts der letzten 30 Jahre zu geniessen, wobei er nacheinander die intrakapsuläre und extrakapsuläre Technik sowie jene durch Phakoemulsifikation erlernte, so dass er mit gründlicher Sachkenntnis die Schwierigkeiten und die Dauer solcher Operationen beurteilen kann. Als Student hatte er das Glück gehabt, Operationen beizuwohnen, die noch mit dem von-Graefe-Messer von Chirurgen der vorigen Generation vorgenommen wurden.

Übersetzung aus dem Französischen ins Deutsche: Karl Bühler 2 Robert Liston (1794-1847), berühmter Chirurg, der als erster Engländer die Anästhesie, vor allem Etherisation, benutzte.

Korrespondenz:

PD Dr. med. X. Martin

Augenarzt und Ophthalmochirurg FMH

64, avenue de Rumine

CH-1005 Lausanne
Obgleich jeder Fall verschieden war und es auch heute noch ist, dauerte eine einfache, von Expertenhand ausgeführte Operation des Katarakts mit dem von-Graefe-Messer kaum mehr als 10 Minuten, die intrakapsuläre etwa 15 Minuten und die extrakapsuläre 20 Minuten.

Die Phakoemulsifikation (Abb. 2) von Charles Kelman [4], 1967 eingeführt, ist erst 20 Jahre später, d.h. seit 1987, die Operation der Wahl geworden. Diese Technik erlaubt es, wohl besser, nicht aber schneller zu operieren und ist vor allem heikler als die früheren Methoden; ferner verlangt sie die Kenntnis und Beherrschung einer sehr komplexen und kostspieligen Technologie. Zudem ist die mittlere Dauer einer Operation durch Phakoemulsifikation länger als diejenige der früheren Methoden und schwankt um die 25 Minuten für einen einfachen Fall und bedeutend länger bei schwierigen Fällen. Natürlich hat jede der aufgeführten Techniken ihre Verfechter, die sich rühmen, schneller und besser als ihre Kollegen zu operieren, und die darauf ausgehen, diese Chirurgie zu denigrieren. Dieser unangenehme Quergang ist übrigens von William Tasman, dem damaligen Vorsitzenden der amerikanischen Akademie für Ophthalmologie in seiner Eröffnungsrede des jährlichen Kongresses dieser Institution im Jahre 1998 gebrandmarkt worden [5].

\section{Abbildung 1}

Operation des Katarakts um 1583 [7] beim Patienten zu Hause ausgeführt. Die Operation, hier durch Reklination, dauerte nur einige Minuten.

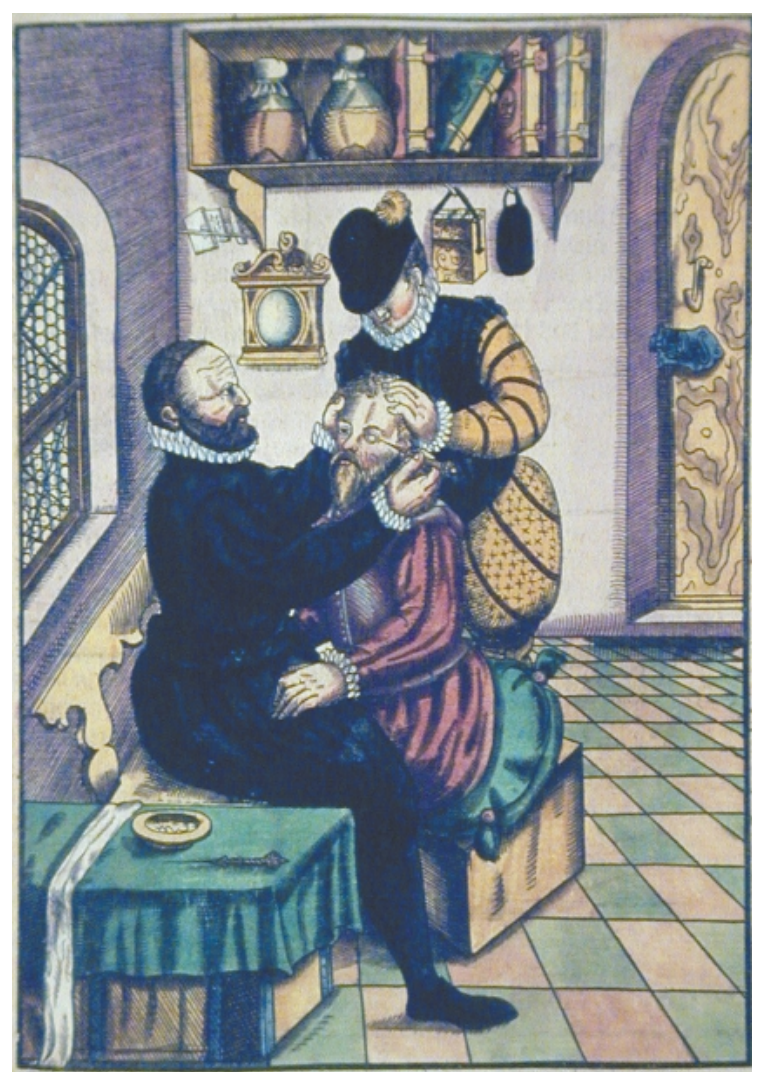




\section{Abbildung 2}

Operation des Katarakts im Jahre 2000 durch Phakoemulsifikation im Operationssaal ausgeführt. Während bei einer Operation des Katarakts die durchschnittliche Besetzung des Operationssaals 60 Minuten ausmacht, beträgt die mittlere Operationszeit bei einem einfachen Katarakt etwa 25 Minuten.

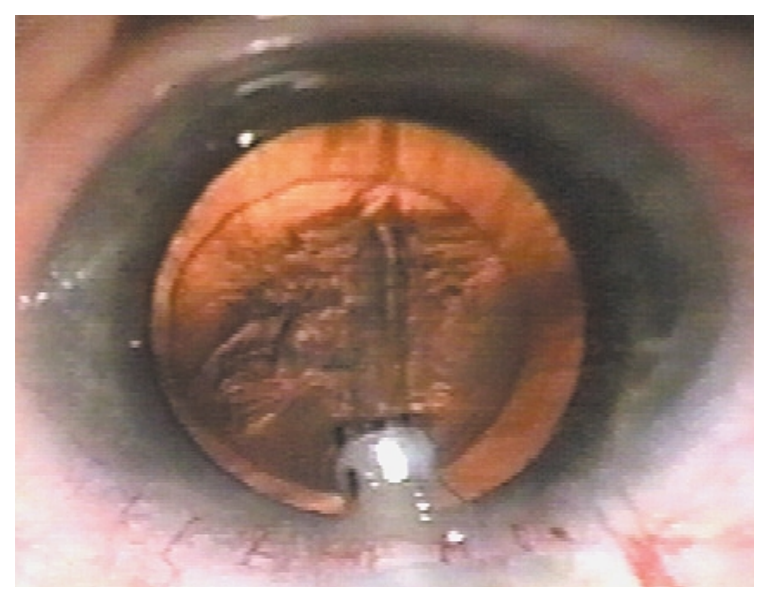

Ähnliche Fakten könnten angeführt und auf das Glaukom bezogen werden, deren Arbeitsmethoden heute heikler und deren Ausführung länger als die frühere sind. Die Thermosklerektomie nach Scheie (10 Minuten), die Trabekulektomie (20 Minuten) und die nicht perforierende tiefe Sklerektomie (25-30 Minuten) erinnern uns deutlich daran.
Es ist freilich zu bedauern, dass Personen sich mit diesem Thema befassen, die gar nichts davon verstehen, wie es auch bedauerlich ist, dass ihre Erklärungen veröffentlicht werden. Sollten diese der Bevölkerung abgegebenen Informationen nicht auf solider Sachkenntnis beruhen? Machen solche Erklärungen nicht jegliche Diskussion zunichte? Das sind Fragen, die man sich mit Recht stellen darf.

\section{Literatur}

1 Tröhler U. L'essor de la chirurgie. In: Grmek MD (Hrsg.). Histoire de la pensée médicale en 0ccident. 3. Du romantisme à la science moderne. Paris: Edition du Seuil; 1999. p. 249.

2 Daviel, J. Sur une nouvelle méthode de guérir la cataracte par l'extraction du cristallin. Mémoire présenté devant l'Académie royale de chirurgie à Paris, le 16 novembre 1752. Mem Acad Roy Chir 1752;2:337-54.

3 Emery JM, McIntyre DJ. Extracapsular cataract surgery. St. Louis: C.V. Mosby Company; 1983. p. 3-6.

4 Kelman CD. Phaco-emulsification and aspiration. A new technique of cataract removal. A preliminary report. Am J Ophthalmol 1967;64:23-35.

5 Tasman, WS. President-elect Address. Final Program. The American Academy of Ophthalmology; 1998. p. 96.

6 Lyons AS, Petrucelli J. Histoire illustrée de la médecine. Paris: Presses de la Renaissance; 1979. p. 517.

7 Bartisch G. Das ist Augendienst. Dresden; 1583. S. 62. 